\title{
【交通物流／Transportation Logistics】
}

\section{新型立体环形交叉口的运行效率研究}

\author{
潘兵宏 ${ }^{1,2}$ ，周廷文 ${ }^{1}$, 温长鹏 ${ }^{1}$ ，单慧敏
}

1) 长安大学公路学院，陕西西安 $710064 ； 2 ）$ 长安大学特殊地区公路工程教育部重点试验室, 陕西西安 710064

摘 要: 常规环形交叉口能够有效避免交通冲突, 但随着机动车数量的增加, 出现了通行能力不足的 问题. 提出一种新型立体环形交叉口，通过设置上下两个环圈来解决环形交叉口通行效率较低的问题。利 用 VISSIM 仿真软件，从通行效率和行车安全方面进行模拟，发现相较于常规环形交叉口，新型立体环形交 叉口使路网的平均延误降低 $91.78 \%$ ，冲突总次数降低 $73.4 \%$; 与简易立交对比发现，新型立体环形交叉 口除东西直行方向的通行效率降低外，其余方向均有所提高. 进一步分析新型环形交叉口的适应交通量表 明, 当各入口的交通量小于 $2000 \mathrm{pcu} / \mathrm{h}$ 时, 立体环形交叉口能有效提高通行能力, 随着右转比例提高, 左 转比例降低，通行效率的提升更加明显. 结合建造成本、占地规模及建造可行性，分析了立体环形交叉口 的适用性. 在交通量需求较大、相交道路没有主次之分、工程预算不足以修建立体交叉以及可利用土地范 围有限等情况下，通过新建或改建成立体环形交叉口可以有效改善路网的运行效率和行车安全.

关键词：交通工程；环形交叉口，VISSIM 仿真；延误；间隙-接受原理；交叉口设计

中图分类号：U491 文献标志码：A doi：10.3724/SP. J. 1249.2020.06630

\section{Operational efficiency of new stereo roundabouts}

\section{PAN Binghong ${ }^{1,2}$, ZHOU Tingwen ${ }^{1}$, WEN Changpeng ${ }^{1}$, and SHAN Huimin ${ }^{1}$}

1) School of Highway, Chang'an University, Xi'an 710064, Shaanxi Province, P. R. China

2) Key Laboratory for Special Area Highway Engineering of Ministry of Education, Chang'an University,

Xi'an 710064, Shaanxi Province, P. R. China

\begin{abstract}
The conventional roundabouts can effectively avoid traffic conflicts. However, as the number of vehicles increases, insufficient capacity begins to appear. To solve this problem, a new type of roundabout is proposed by setting lane roundabouts on two levels with right-hand turning bypasses. By using VISSIM software, the stereo roundabouts are compared with the actual conventional roundabouts and simple interchanges. The evaluation results show that compared with the conventional roundabouts, the stereo roundabout reduces the average delay of the road network by $91.78 \%$, the total number of conflicts decreases by $73.4 \%$. Compared with the simple interchange, the efficiency of the stereo roundabouts is improved in all directions except the east-west direction. Through further analysis of the traffic volume that stereo roundabouts can adapt to, it shows that the stereo roundabout can effectively improve the traffic capacity when the traffic volume at each entrance is less than $2000 \mathrm{pcu} / \mathrm{h}$. As the right turn ratio increases and the left turn ratio decreases, the improvement of traffic efficiency is more obvious. Besides, the applicability of stereo roundabout is also analyzed based on the construction cost, floor space and construction
\end{abstract}

Received: 2019-10-07; Accepted: 2019-12-06

Foundation: Livelihood Scientific Special Foundation of Hebei Province (19275601D)

Corresponding author: Associate professor PAN Binghong. E-mail: 409291838@ qq.com

Citation: PAN Binghong, ZHOU Tingwen, WEN Changpeng, et al. Operational efficiency of new stereo roundabouts [J]. Journal of Shenzhen University Science and Engineering, 2020, 37(6): 630-636. (in Chinese)

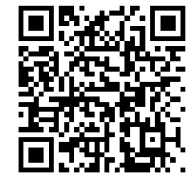


feasibility. Under certain circumstances with large traffic demand, no distinction between primary and secondary roads, insufficient project budget for the construction of interchange, and limited land available, the operation efficiency and traffic safety of road network can be effectively improved by building or rebuilding stereo roundabouts.

Key words: traffic engineering; roundabout; VISSIM simulation; delay; gap-acceptance theory; intersection design

环形交叉可以有效避免交叉口处的交通冲突， 由于中国早期机动车保有量较少，在相当长的一个 时期内，环形交叉口设计对提高路口通行能力起到 重要作用. 但随着经济的快速增长，城市机动车保 有量急速上升, 交通需求急剧增长, 环形交叉口通 行能力不足的弊端逐渐显露，出现大量环形交叉口 长时间交通拥堵的现象.

针对环形交叉口通行能力不足的问题，已有研 究提出诸多解决措施. 杨锦冬等 ${ }^{[1]}$ 通过对环形交叉 口交通特征与交通问题深人研究后，提出 3 种信号 控制方法，以适应不同规模的环形交叉口. 马新露 等 ${ }^{[2]}$ 研究传统信号控制四路形交叉口环内车辆间相 互干扰对交叉口延误的影响，提出多进口协同方形 的信号控制方法. 郑元勋等 ${ }^{[3]}$ 针对左转车流较多的 环形交叉口，提出部分左转立体式环形交叉口的概 念. 张超等 ${ }^{[4]}$ 为减少交织区的车流量, 并缩短左转 车辆的绕行距离, 提出卫星岛式环形交叉口. MEHMOOD 等 ${ }^{[5]}$ 研究不同渠化方法及几何特性情况 下，环形交叉口的运行效率. YANG 等 ${ }^{[6]}$ 提出一种 新型环岛控制方法，通过在环岛环流区和人口处设 置信号灯，实现双重信号控制以提升环形交叉口的 通行效率. FORTUIJN 等 ${ }^{[7]}$ 针对环形交叉口通行能 力不足的问题，提出浴轮环形交叉口的设计理念， 已在欧洲许多国家得到应用. Hamburger、dumbbell、turbo-square 及 dog-bone 等多种新型环形交叉 口也相继提出, 用来改善现有环形交叉口通行能力 不足的问题 ${ }^{[8-9]}$.

以上研究主要从信号配时的优化方面改善环形 交叉口的通行状况，还有少部分研究从渠化方面提 出一些新型环形交叉口形式. 其中, 浴轮环形交叉 口在国外应用最为广泛, 目前已经建成的浴轮环形 交叉口达到 480 座 $^{[10]}$, 但相关研究表明, 浴轮环形 交叉口的提升效果主要集中在道路的安全方面，在 通行能力方面提升尚不显著 ${ }^{[11-12]}$. 本研究在分析国 内外环形交叉口特点的基础上，提出一种新型立体 环形交叉口，以期同时提升道路通行效率及行车安 全，并分别对比新型环形交叉口与传统环形交叉口 以及立体交叉口的运行效率.

\section{1 交通组织}

\section{1 交通组织形式}

常规环形交叉口环道上的车辆具有优先通行 权, 人环的车辆需要在人口处排队等待, 当出现可 利用的间隙时, 方可驶人环道. 左转车辆由人口处 的内侧车道进入环道，在交织区完成换道行为后进 人内侧环道, 当需要驶出环道时, 仍需通过交织行 为完成车道变换后驶出. 直行和右转车辆由人口处 的外侧车道进入环道, 直行车辆一般在环道的外侧 车道上完成直行, 右转车辆则通过利用环道与路缘 带之间的间隙驶出环道.

立体环形交叉口的几何构造如图 1, 该环形交 叉口由上下两个环圈组成. 上部环圈供东西直行及 左转车辆行驶, 下部环圈供南北直行及左转车辆行 驶. 人口处的左侧车道为直行和左转车道, 右侧车 道为右转专用车道, 与左转和直行车道之间设置物 理分隔设施. 左转和直行的车辆需要通过驶人环道 完成直行和左转, 右转车辆直接通过右转专用车道 完成右转. 当右转车辆因操作失误而驶人左侧车道 时, 仍可通过驶入环圈后的第 1 个出口完成右转.

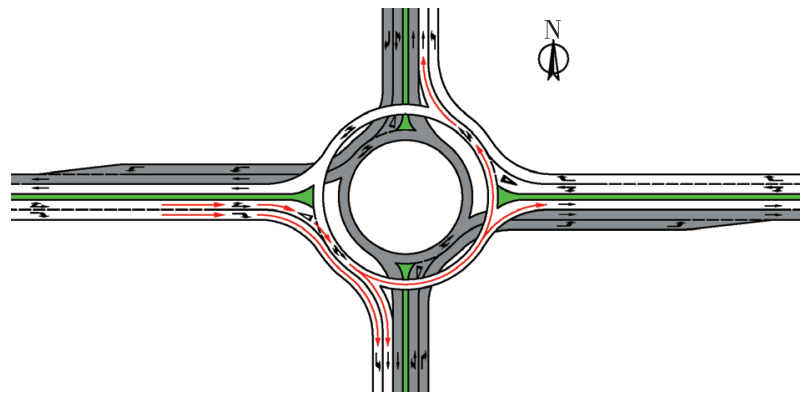

图 1 立体环形交叉口

Fig. 1 A stereo roundabout

\section{2 交通组织对比分析}

以双向 4 车道的十字交叉口为例, 立体环形交 叉口与常规环形交叉口相比, 具有以下特点.

1) 由环形交叉口交错点分布（图 2) 可知，立 体环形交叉口的合流点和分流点数量没有变化, 但 冲突点由 8 个减少至 0 , 同时将 4 个合流点从环道 
转移到出口道上, 极大提升了交叉口的通行效率和 行车安全.

2) 右转车道与左转、直行车道之间有硬隔离, 两条车道上的车辆互不影响, 有利于行车安全, 同 时也提高了右转车辆的通行效率.

3）每个人口处的车辆只受到对向左转车流的 影响, 提高车辆驶入环道的概率.

4) 左转和直行车辆在环道上行驶时, 只存在 跟驰行为, 不存在交织行为, 缩短车辆的行驶时 间, 提高行车安全.

5）每个环圈上只有两个方向直行和左转的车 辆驶人, 减少穿插和交织行为, 提高了通行效率, 降低环道上产生交通事故的概率.

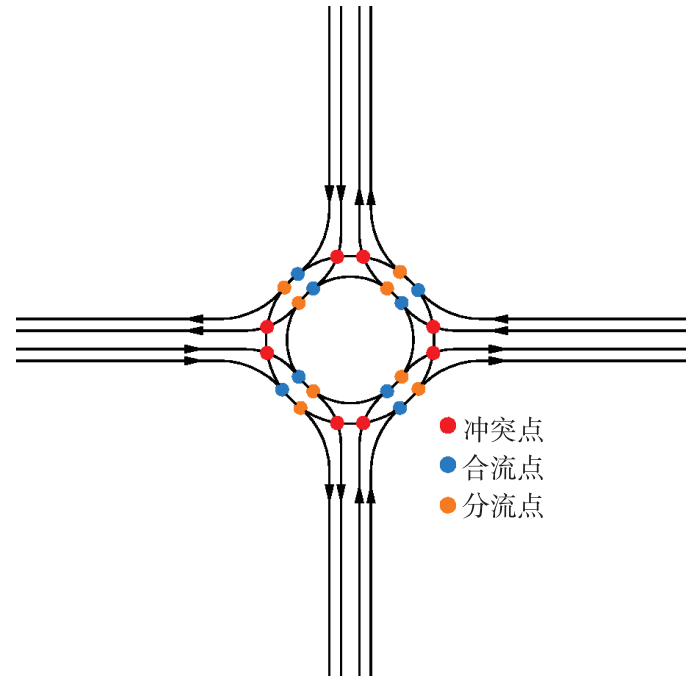

（a）常规环形交叉口

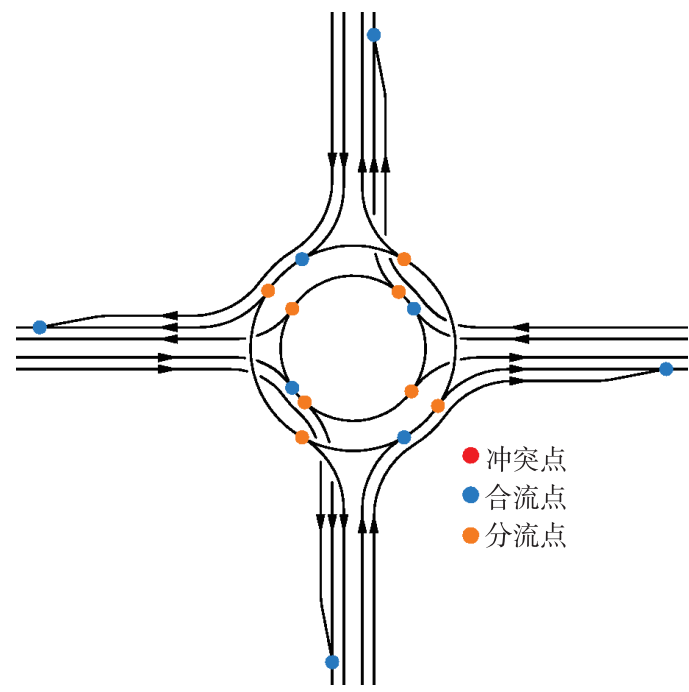

（b）立体环形交叉口

图 2 环形交叉口交错点分布

Fig. 2 Conflict spots of roundabout

\section{2 立体环形交叉口的通行能力分析}

目前环形交叉口通行能力的计算模型包括: (1) 交织理论模型, 即通过环形交叉口环道的交织段所 能通过的最大交织流量反映环形交叉口的通行能 力; (2) 间隙-接受理论模型, 根据进口车道能进人 环形交叉口的最大车流量计算环形交叉口的通行能 力; (3) 经验回归模型. 许多国家通过回归分析的 方法建立环道车流量与通行能力之间的关系模型.

立体环形交叉口的特点包括：(1) 右转车道与 左转、直行车道之间有硬隔离, 两条车道上的车辆互 不影响; (2) 环道上的车辆具有优先通行权, 人环 车辆需给环道上车辆让行. 因此, 本研究基于间隙接受理论, 建立立体环形交叉口的通行能力模型.

\section{1 左直行车道的通行能力}

环形交叉口的左侧进口车道提供车辆直行与左 转, 车辆需要在人环处等待合适的间隙才能驶人环 道，其车流将与环道上的车流产生穿插现象. 由于 环道上行驶的车辆不存在超车行为, 因此, 可认为 环道上车辆的车头时距服从移位负指数分布.

根据项乔军等 ${ }^{[13]}$ 对环形交叉口通行能力模型 的研究, 左直行车道的通行能力可表示为

$$
\begin{aligned}
& C_{\mathrm{TL}}=3600 \sum_{k=1}^{\infty} p^{k} k q=3600 \frac{T q \mathrm{e}^{-\lambda\left(t_{\mathrm{c}}-t_{\mathrm{m}}\right)}}{1-\mathrm{e}^{-\lambda t_{\mathrm{f}}}} \\
& \lambda=T q /\left(1-t_{m} q\right)
\end{aligned}
$$

其中, $C_{\mathrm{TL}}$ 为左直行车道的通行能力 (单位: $\mathrm{pcu} / \mathrm{h}$ ); $T$ 为车头时距大于最小时距的自由流比例; $p^{k}$ 为允 许 $k$ 辆车进入环形交叉口的概率; $k$ 为进人环形交叉 口的车辆数 (单位: pcu); $\lambda$ 为衰减常量; $q$ 为环形 车流量 (单位: $\mathrm{pcu} / \mathrm{s}$ ); $t_{\mathrm{c}}$ 为临界间隙 (单位: $\mathrm{s}$ ) ; $t_{\mathrm{m}}$ 为最小时距 (单位: $\mathrm{s}$ ); $t_{\mathrm{f}}$ 为随车时距 (单位: $\mathrm{s}$ ).

\section{2 右转车道通行能力}

由于立体环形交叉口的上环与下环均为右转方 向车辆提供专用车道, 且渠化程度较高, 不会与其 他方向的行驶车辆产生冲突. 因此, 在计算右转车 道通行能力时, 可以采用停车线法计算右转专用车 道的通行能力，计算方法为

$$
C_{\mathrm{R}}=\frac{3600}{t_{\mathrm{r}}}
$$

其中, $C_{\mathrm{R}}$ 为右转专用车道的通行能力 (单位: $\mathrm{pcu} / \mathrm{h}) ; t_{\mathrm{r}}$ 为前后两右转车辆连续驶过停车线断面 的间隔时间 (单位: $s$ ). 根据观测, 当大小车各占 
$1 / 2$ 时, $t_{\mathrm{r}}$ 的平均值约为 $4.5 \mathrm{~s}$; 单纯为小车时, $t_{\mathrm{r}}$ 的 平均值为 $3.0 \sim 3.6 \mathrm{~s}^{[14]}$.

\section{3 立体环形交叉口总通行能力}

根据左直行车道的通行能力计算方法可知, 人 口处左直行车道的通行能力与环道车流量有关. 当 环道车流量较低时, 各人口处左直行车道的通行能 力较高, 随着人口处车辆进入环道, 导致环道的车 流量逐渐增加, 从而又使得人口处左直行车道的通 行能力降低. 因此, 立体环形交叉口总通行能力不 能通过各人口的通行能力相加得到. 当立体环形交 叉口各人口左直行车道的车流量逐渐增加至通行能 力时, 人口处的流量不能再增加, 整个环形交叉口 处于稳定状态, 此时再加上右转专用车道的通行能 力, 即为该环形交叉口的总通行能力.

计算人口处左直行车道的通行能力时, 可采用 迭代的方法, 具体步骤为: (1) 通过实际调查, 确 定出环形交叉口的临界间隙、最小时距、随车时 距、人口流量及转向比例; (2) 以实际人口流量为 初始值计算环道流量; (3) 将环道流量带人式 (1), 计算出人口处左直行车道的通行能力; (4) 以计算 出的人口处左直行车道通行能力作为各人口流量, 重新计算环道流量; (5) 重新计算各人口处左直行 车道的通行能力; (6) 重复步骤(4)和 (5), 当前后两 次的通行能力值差在小范围内变化时, 停止迭代.

此时, 各人口的通行能力值达到稳定, 立体环 形交叉口总通行能力为各人口通行能力之和. 根据 薄春宇等 ${ }^{[15]}$ 对环形交叉口交通流特征参数的研究 结果, 在各进口车流量的左右转向比例均为 $15 \%$ 时, 得到双向 4 车道常规环形交叉口的基本通行能力为 $4100 \mathrm{pcu} / \mathrm{h}$ ，将其计算所采用的交通流特征参数代 人立体环形交叉口计算模型, 得到基本通行能力为 $9312 \mathrm{pcu} / \mathrm{h}$, 与常规环形交叉口相比提高 $127 \%$.

\section{3 交通仿真实验}

\section{1 实例选取}

为验证立体环形交叉口在通行效率和行车安全 方面的提升效果，拟选取一个常规环形交叉口和一 个简易立体交叉口进行交通量调查，将其交通运行 状况在 VISSIM 软件中还原, 通过在原占地规模的 基础上建立相应的立体环形交叉口模型，对比两者 的通行效率和行车安全性.

选取的常规环形交叉口位于中国武汉市江汉 区，简易立交位于西安市雁塔区，为双层式环形立
体交叉. 根据高德交通 ${ }^{[16]}$ 对城市交通拥堵延时的 分析发现，武汉和西安市在 18:00-19:00 的拥堵延 时指数最高, 因此, 选取该时段对交叉口的交通量 和行驶速度进行采集.

常规环形交叉口各人口均为双向 4 车道, 行车 道宽度为 $3.5 \mathrm{~m}$, 有 3 条环道，宽度均为 $4.0 \mathrm{~m}$, 环岛直径为 $40 \mathrm{~m}$. 经实际调查, 东西走向实测车速 为小客车 $55 \sim 60 \mathrm{~km} / \mathrm{h}$, 公交车 $40 \sim 50 \mathrm{~km} / \mathrm{h}$; 南 北走向的实测车速为小客车 $50 \sim 60 \mathrm{~km} / \mathrm{h}$, 公交车 $40 \sim 50 \mathrm{~km} / \mathrm{h}$. 环道上的实测车速为 $15 \sim 20 \mathrm{~km} / \mathrm{h}$.

简易立交连接东西走向的二环南路和南北走向 的雁塔北路. 二环南路是双向 6 车道的城市快速 路, 两侧均设置有辅路. 雁塔北路是双向 6 车道的 城市主干路. 二环南路右转和左转方向的车辆均通 过两侧辅路驶人雁塔北路的环岛完成转向. 经实际 调查, 行车道宽度均为 $3.5 \mathrm{~m}$, 环道宽度为 $4.0 \mathrm{~m}$. 东西走向实测车速为小客车 $60 \sim 70 \mathrm{~km} / \mathrm{h}$ 、公交车 $55 \sim 60 \mathrm{~km} / \mathrm{h}$. 南北走向实测车速为小客车 $45 \sim 50$ $\mathrm{km} / \mathrm{h}$ 、公交车 $35 \sim 40 \mathrm{~km} / \mathrm{h}$. 环道上的实测车速为 $15 \sim 20 \mathrm{~km} / \mathrm{h}$.

\section{2 模型校准}

通过采集现场的交通量、行车速度以及几何参 数对模型进行校准, 以提高 VISSIM 仿真实验的精 确度. 将实测小客车与公交车的比例及速度分布输 人 VISSIM 中进行仿真模拟，使用平均绝对相对误 差 $^{[17]}$ ( mean absolute percent error, MAPE) 评价其仿 真效果的准确性. MAPE 值越小, 仿真可信度越 高. MAPE 值为

$$
\text { MAPE }=\left|\frac{C_{\mathrm{s}}^{i}-C_{\mathrm{f}}^{i}}{C_{\mathrm{f}}^{i}}\right| \times 100 \%
$$

其中, $C_{\mathrm{s}}^{i}$ 为仿真模拟得出的交通量 (单位: $\mathrm{pcu} /$ $\mathrm{h}) ; C_{\mathrm{f}}^{i}$ 为调查实测得到的交通量 (单位: $\mathrm{pcu} / \mathrm{h}$ ); $i$ 为实际调查编号或与之对应的仿真模型编号. 通过 交通仿真得到的结果如表 1. 可见，常规环形交叉 口和简易立交的 MAPE 值分别为 $9.48 \%$ 和 $11.40 \%$ ，在工程可接受范围内 ${ }^{[18]}$.

表 1 VISSIM 仿真校准结果

Table 1 VISSIM simulation calibration results

\begin{tabular}{cccc}
\hline 交叉口类型 & $\begin{array}{c}\text { 实测交通量/ } \\
\left(\mathrm{pcu} \cdot \mathrm{h}^{-1}\right)\end{array}$ & $\begin{array}{c}\text { 仿真交通量/ } \\
\left(\mathrm{pcu} \cdot \mathrm{h}^{-1}\right)\end{array}$ & $\mathrm{MAPE} / \%$ \\
\hline 常规环形 & 3910 & 3539 & 9.48 \\
简易立交 & 13810 & 12235 & 11.40 \\
\hline
\end{tabular}




\section{4 仿真实验数据分析}

\section{1 立体环形交叉口与常规环形交叉口对比}

通过 VISSIM 仿真获得交叉口的平均延误，并 应用美国联邦公路局开发的交通冲突评价模型 ( surrogate safety assessment model, SSAM) 分析仿真获得 的车辆轨迹文件, 得到常规环形交叉口与立体环形 交叉口在实测交通量条件下的通行效率和安全评价 结果，见表 2. 可见，与常规环形交叉口相比，立 体环形交叉口在平均延误、追尾次数和变道次数方 面均有大幅度改善, 交叉口的通行效率和行车安全 得到显著提升.

\section{表 2 通行效率与行车安全对比分析}

Table 2 Operational efficiency and safety performance of conventional roundabout and stereo roundabout

\begin{tabular}{cccc}
\hline 交叉口类型 & 平均延误/s & 追尾次数/次 & 变道次数/次 \\
\hline 常规环形 & 73 & 386 & 99 \\
立体环形 & 6 & 96 & 33 \\
\hline 降低比率 $/ \%$ & 91.78 & 75.13 & 66.66 \\
\hline
\end{tabular}

该常规环形交叉口的左转车辆占比为 $16.72 \%$, 人口处的车辆会受到对向左转、左侧人口直行与左 转 3 条车流的影响，而立体环形交叉口人口处的车 辆仅受到对向左转车辆的影响. 与常规环形交叉口 相比, 立体环形交叉口人口处的车辆受环道车流的 影响较小，车辆能够快速驶入环道，显著提高了交 叉口的通行效率.

从行车安全方面分析，立体环形交叉口只有 1 条环道, 车辆在环道行驶时仅存在跟驰行为, 减少 了车辆变道次数. 立体环形交叉口每个环圈仅有 2 个人口，环道影响人环的车辆数比常规环形交叉口 少, 且立体环形交叉口设置了物理隔离的右转专用 车道，右转车辆不会与环道上的车辆产生穿插现 象, 使立体环形交叉口的追尾和变道次数大幅下降.

\section{2 立体环形交叉口与简易立交对比}

考虑到简易立交结构的复杂性，采用路网平均 延误值并不能体现两种交叉口在运行效率方面的差 异. 因此, 采用各行驶路径车辆的平均延误进行运 行效率的对比分析, 结果如表 3. 其中, E、S、W 及 $\mathrm{N}$ 分别代表东南西北 4 个方向; $\mathrm{W}-\mathrm{S}$ 代表由西 向南行驶的车辆.

通过对比简易立交和立体环形交叉口各车辆行 驶路径的延误发现，立体环形交叉口在南北走向各
路径上的行车延误均有大幅下降, 其中, 右转和直 行降低比率较大, 平均降低达 $82 \%$; 左转车辆的延 误降低率则相对较低为 $69 \%$ 。两种交叉口南北走向 的车辆均需要通过环岛完成直行和左转, 但与简易 立交相比，立体环形交叉口减少了东西走向右转和 左转车辆的影响, 使得立体环形交叉口南北走向的 通行效率提升较大.

表 3 各行驶路径延误对比分析

Table 3 Comparative analysis of delay in each driving route

\begin{tabular}{ccccccc}
\hline \multirow{2}{*}{ 交叉口类型 } & \multicolumn{6}{c}{ 行驶路径延误/s } \\
\cline { 2 - 7 } & $\mathrm{W}-\mathrm{S}$ & $\mathrm{W}-\mathrm{E}$ & $\mathrm{W}-\mathrm{N}$ & $\mathrm{E}-\mathrm{N}$ & $\mathrm{E}-\mathrm{W}$ & $\mathrm{E}-\mathrm{S}$ \\
\hline 简易立交 & 132 & 29 & 150 & 56 & 23 & 75 \\
立体环形 & 49 & 89 & 71 & 21 & 63 & 32 \\
\hline 降低比率/\% & 63 & -206 & 53 & 63 & -174 & 57 \\
\hline \hline 交叉口类型 & \multicolumn{7}{c}{ 行驶路径延误/s } \\
\cline { 2 - 7 } & $\mathrm{S}-\mathrm{E}$ & $\mathrm{S}-\mathrm{N}$ & $\mathrm{S}-\mathrm{W}$ & $\mathrm{N}-\mathrm{W}$ & $\mathrm{N}-\mathrm{S}$ & $\mathrm{N}-\mathrm{E}$ \\
\hline 简易立交 & 47 & 151 & 76 & 35 & 56 & 69 \\
立体环形 & 9 & 15 & 22 & 5 & 16 & 23 \\
\hline 降低比率/\% & 81 & 90 & 71 & 86 & 71 & 67 \\
\hline
\end{tabular}

立体环形交叉口在东西走向右转和左转方向的 行车延误降低比率均达 $50 \%$ 以上，在直行方向的行 车延误平均增加 $190 \%$ 。 由于简易立交东西走向的 直行车辆不受南北走向车辆的影响，导致其延误低 于立体环形交叉口. 但其左转和右转车辆均需通过 两侧辅道驶人环岛才能完成转向行驶，因此，会受 到环道上车辆的影响, 使其行车延误均比立体环形 交叉口要高.

由以上结果可见，立体环形交叉口与简易立交 相比，虽然东西直行方向的通行效率有所下降，但 其他方向的通行效率均有提升，且达到 $50 \%$ 以上.

\section{3 适用交通量分析}

4. 2 节仅分析 1 种交通状况下的通行效率，结 果具有一定局限性. 为了验证立体环形交叉口所能 适应的交通量，以双向 4 车道立体环形交叉口为基 础模型，在 VISSIM 模型中进行不同交通状况的模 拟仿真，以确定立体环形交叉口的适用性.

影响环形交叉口通行效率的因素包括小汽车与 公交车的比例、各人口交通量及转向比例等. 本研 究选取交通量和转向比例进行适用性分析, 其中, 交通量以计算出的基本通行能力 $9312 \mathrm{pcu} / \mathrm{h}$ 为基 准, 各人口处的取值为 $1000 \sim 2400 \mathrm{pcu} / \mathrm{h}$, 将其 
分为 8 组, 梯度为 $200 \mathrm{pcu} / \mathrm{h}$. 由于立体环形交叉 口设置了右转专用车道, 左转和直行车辆需要在环 道上绕圈行驶, 且行驶距离较长, 对环形交叉口的 延误影响较大. 因此, 将人口处右转交通量与该人 口处总交通量的比值分为 $20 \% 、 30 \%$ 及 $40 \%$ 三组, 每组右转比例对应 3 种左转和直行的组合, 每种组 合中左转比例取 $20 \% \sim 40 \%$, 以 $10 \%$ 的梯度增加.

根据仿真实验得到立体环形交叉口的平均延 误, 如图 3. 可见, 当各方向转向比例固定时, 随 着交通量的增加，立体环形交叉口的平均延误逐渐 上升. 当各人口的交通量超过 $2000 \mathrm{pcu} / \mathrm{h}$ 时，平 均延误增长变缓, 立体环形交叉口出现拥堵, 车辆 平均延误约达 $120 \mathrm{~s}$, 交叉口通行效率较低. 当右 转比例固定时, 同一交通量下随着左转车辆比例的 增加，立体环形交叉口的平均延误也逐渐上升，在 右转比例为 $20 \%$ 时, 随着左转比例的增加, 其平均 延误上升幅度相比其他右转比例 (30\% 和 40\%) 要 大; 当左转比例和交通量固定时, 随着右转车辆比

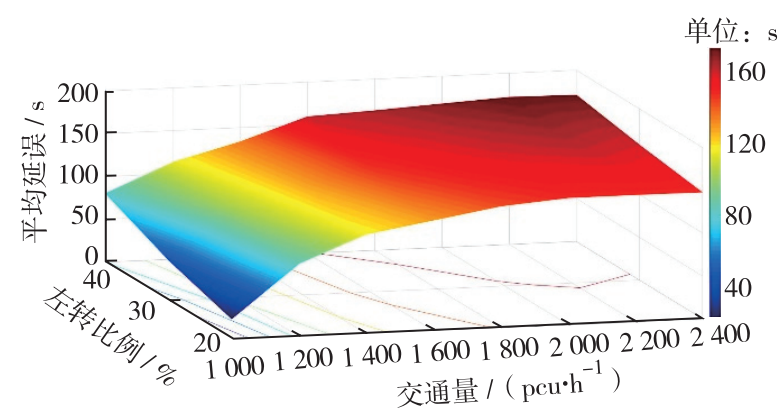

(a) 右转比例为 $20 \%$

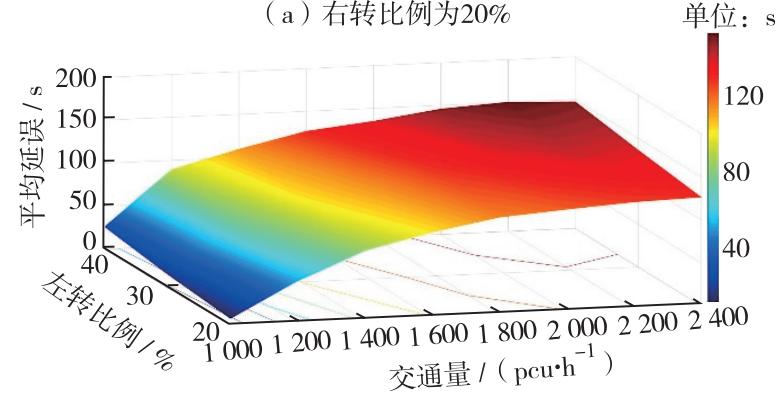

(b) 右转比例为 $30 \%$

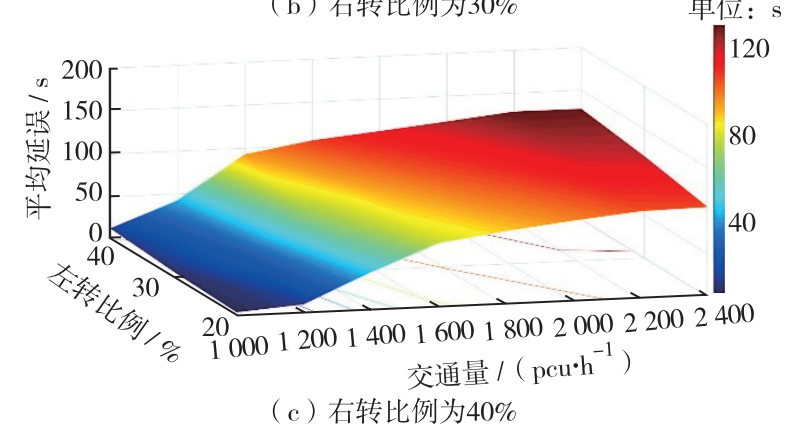

图 3 立体环形交叉口平均延误

Fig. 3 Average delay of stereo roundabout
例增加，立体环形交叉口的平均延误逐渐降低.

由以上结果可知, 对于双向 4 车道十字立体环 形交叉口，其所能适应的各人口处交通量为 2000 $\mathrm{pcu} / \mathrm{h}$, 总交通量为 $8000 \mathrm{pcu} / \mathrm{h}$. 随着交叉口右转 车辆比例的增加, 左转车辆比例减少, 立体环形交 叉口能适应的交通量略有提升.

\section{5 适用性分析}

立体环形交叉口通过设置上下环圈，并在右转 车道处设置物理隔离达到提升通行效率的目的，建 造时可以通过改变环圈直径大小控制占地范围. 由 于立体环形交叉口在空间上对道路进行分离，使行 人和非机动车过街时仅需穿越一侧的行车道. 立体 环形交叉口比常规环形交叉口的造价成本高; 但与 立体交叉口相比，由于其仅需设置上下环圈，且可 以通过控制环圈直径减少占地，因此，总造价比立 体交叉口低. 该立体环形交叉口的上部环圈直径比 下部环圈大，在建造时根据上跨行车道的宽度选择 合适跨径的桥梁，并通过压缩中央分隔带宽度，选 用悬挑梁实现上部环圈出人口处桥墩的架设.

因此，在应用立体环形交叉口时，需充分考虑 交叉口的交通量、地理位置及工程预算等因素. 对 于交通量需求较大、相交道路没有主次之分、工程 预算不足，以及可利用土地范围不足以修建立体交 叉的城市道路交叉口，适于新建或改建成立体环形 交叉口以提升通行效率和行车安全.

\section{结 语}

为提高传统十字环形交叉口的通行效率和行车 安全, 提出一种新型立体环形交叉口. 通过设置上 下环圈，将 4 个方向的车流从空间上进行分离，达 到提升通行效率和行车安全的效果. 分析立体环形 交叉口车辆行驶特征，建立立体环形交叉口通行能 力计算模型，结果表明，立体环形交叉口的基本通 行能力比常规环形交叉口提高 $127 \%$ 。交通仿真对 比分析结果表明，与常规环形交叉口相比，立体环 形交叉口使路网平均延误、追尾次数及变道次数分 别降低 $91.78 \%$ 、 $75.13 \%$ 及 $66.66 \%$ ，有效提高了 交叉口的通行效率和行车安全. 与简易立交相比, 立体环形交叉口在直行方向的通行效率有所降低, 但在其他各行驶方向的通行效率均有提升，达到 $50 \%$ 以上. 在适用的交通量范围以及不同转向比例 
条件下，对双向 4 车道立体环形交叉口的运行效率 进行分析，发现当交叉口各人口的交通量在 2000 $\mathrm{pcu} / \mathrm{h}$ 以下时，设置立体环形交叉口能有效改善道 路运行效率，当右转车辆比例较高，左转车辆比例 较少时，运行效率改善效果更好. 本研究将交叉口 置于一个孤立的点，后续工作可考虑基于周边路网 来更精确评估立体环形交叉口的运行效率.

基金项目: 河北省科学技术厅民生科技专项基金资助项目 (19275601D)

作者简介：潘兵宏 (1974一)，长安大学副教授、博士. 研究方向 互通式立交工程与道路安全. E-mail：409291838@ qq.com

引文：潘兵宏，周廷文，温长鹏，等，新型立体环形交叉口的 运行效率研究 $[\mathrm{J}]$. 深圳大学学报理工版, 2020, 37 (6) : 630-636

\section{参考文献 / References:}

[ 1 ] 杨锦冬，杨晓光，彭国雄. 环形交叉口交通控制模式 研究 $[\mathrm{J}]$. 公路交通科技，2000，17(3)：47-51.

YANG Jindong, YANG Xiaoguang, PENG Guoxiong. Mode of traffic control in ring-intersection $[\mathrm{J}]$. Journal of Highway and Transportation Research and Development, 2000, 17(3) : 47-51. (in Chinese)

[2] 马新露, 杨兴清. 信号控制四路环形交叉口多进口协 同放行方法 $[\mathrm{J}]$. 交通运输系统工程与信息, 2018, 18 (4) : 69-75.

MA Xinlu, YANG Xingqing. Multi-approach collaborative release method of the signalized four-leg roundabout $[\mathrm{J}]$. Journal of Transportation Systems Engineering and Information Technology, 2018, 18(4) : 69-75. (in Chinese)

[3] 郑元勋, 甘 露. 左转立交式环形交叉口概念设计研 究 $[\mathrm{J}]$. 河南理工大学学报自然科学版, 2017, 36(5): 113-120.

ZHENG Yuanxun, GAN Lu. Conceptual design on roundabout of turn left lane interchange $[\mathrm{J}]$. Journal of Henan Polytechnic University Natural Science, 2017, 36 (5) : 113-120. (in Chinese)

[4] 张 超, 郑元勋. 卫星岛式环形交叉口的概念设计 $[\mathrm{J}]$. 公路, 2013(9): 49-52.

ZHANG Chao, ZHENG Yuanxun. The conceptual design of satellite island roundabout $[\mathrm{J}]$. Highway, 2013(9): 49-52. (in Chinese)

[ 5 ] MEHMOOD A, EASA S M. Optimizing geometric design of roundabouts: multi-objective analysis $[\mathrm{J}]$. Canadian Journal of Civil Engineering, 2006, 33(1) : 29-40.

[ 6 ] YANG Xiaoguang, LI Xiugang, XUE Kun. A new trafficsignal control for modern roundabouts: method and application $[\mathrm{J}]$. IEEE Transactions on Intelligent Transportation Systems, 2004, 5(4) : 282-287.

[ 7 ] FORTUIJN L G H. Turbo roundabouts $[\mathrm{J}]$. Transportation Research Record: Journal of the Transportation Research Board, 2009, 2096(1) : 16-24.
[ 8 ] BROWN M. The design of roundabouts [M ]. London: Her Majesty Stationary Office, 1995.

[ 9 ] TOLLAZZI T. Alternative types of roundabouts [ M ]. Heidelberg, Germany: Springer, 2015.

[10] DŽAMBAS T, AHAC S, DRAGČEVIĆ V. Design of turbo roundabouts based on the rules of vehicle movement geometry $[\mathrm{J}]$. Journal of Transportation Engineering, $2016,143(7): 7-10$.

[11] MAURO R, CATTANI M. Potential accident rate of turboroundabouts $[\mathrm{C}] / /$ Proceedings of the 4 th International Symposium on Highway Geometric Design. Valencia, Spain: Transportation Research Board of the National Academies, 2010: 4-16.

[12 ] MAURO R, CATTANI M, GUERRIERI M. Evaluation of the safety performance of turbo roundabouts by means of a potential accident rate model $[\mathrm{J}]$. The Baltic Journal of Road and Bridge Engineering, 2015, 10(1) : 28-38.

[13] 项乔君, 王 炜, 陈 冰, 等. 环形交叉口通行能力理 论模型研究 $[\mathrm{J}]$. 中国公路学报, 1999, 12(4): 72-75.

XIANG Qiaojun, WANG Wei, CHEN Bing, et al. A study of the model construct ion for roundabout's capacity [J] . China Journal of Highway and Transport, 1999, 12 (4) : 72-75. (in Chinese)

[14] 徐吉谦, 陈学武. 交通工程总论 $[M]$. 北京：人民交通 出版社, 2015.

XU Jiqian, CHEN Xuewu. Fundamentals of traffic engineering $[\mathrm{M}]$. Beijing: China Communications Press, 2015. (in Chinese)

[15] 薄春宇, 崔海梁, 王 炜, 等. 环形交叉口服务通行能 力研究 $[\mathrm{J}]$. 华中科技大学学报自然科学版, 2002, 30 (5) : 47-49.

BO Chunyu, CUI Hailiang, WANG Wei, et al. A study on service capacity of roundabout $[\mathrm{J}]$. Journal of Huazhong University of Science and Technology Natural Science Edition, 2002, 30(5): 47-49. (in Chinese)

[16] AutoNavi Traffic Big-data. Wuhan realtime traffic congestion delay index $[\mathrm{DB} / \mathrm{OL}]$. (2019-08-25 ) [2019-0902 . https : //trp. autonavi. com/detail. do? city = 420100.

[17] 杨文臣, 张 轮, 王 铮, 等. 基于黄金分割遗传算法 的 VISSIM 仿真模型参数校正 $[\mathrm{J}]$. 华东交通大学学 报, 2017, 34(3) : 95-102.

YANG Wenchen, ZHANG Lun, WANG Zheng, et al. Parameter calibration of VISSIM simulation model based on golden-ratio genetic algorithm $[\mathrm{J}]$. Journal of East China Jiaotong University, 2017, 34(3) : 95-102. (in Chinese)

[18 ] 孙 剑. 微观交通仿真分析指南 $[\mathrm{M}]$. 上海：同济大 学出版社, 2014

SUN Jian. Guideline for microscopic traffic simulation analysis [M]. Shanghai: Tongji University Press, 2014. (in Chinese)

【中文责编：方 圆；英文责编：淡 紫】 